\title{
Osteosarcoma condroblástico maxilar: Presentación de un caso
}

\section{Chondroblastic osteosarcoma of the maxilla: A case report}

\author{
Álvarez Martínez E*, Castro Espitia L**, Ardila Medina CM***
}

\section{RESUMEN}

Los osteosarcomas son neoplasmas caracterizados por la presencia de células mesenquimales que producen tejido osteoide. Las manifestaciones clínicas en la cavidad bucal comprenden dolor, inflamación, desplazamiento de los dientes, espasmos, parestesias y obstrucción nasal. Este artículo presenta un osteosarcoma condroblástico avanzado en el maxilar superior. El examen histológico evidenció proliferación neoplásica extensamente cartilaginosa, con áreas pequeñas en la que se identificó formación osteoide y hueso no laminar, rodeado por células neoplásicas. El tratamiento consistió en maxilectomía y vaciamiento bilateral de cuello. Además, se colocó férula quirúrgica obturadora con el fin de sellar el defecto de la comunicación oronasosinusal. Aun cuando el osteosarcoma de los maxilares presenta un mejor pronóstico que el osteosarcoma de los huesos largos, los pacientes con osteosarcoma maxilar pueden presentar tumores avanzados, principalmente cuando no se realiza el diagnóstico adecuado.

Palabras clave: Osteosarcoma, sarcoma osteogénico, neoplasia ósea.

\section{SUMMARY}

Osteosarcomas are neoplasms characterized by the presence of mesenchymal cells that produce osteoid. The clinical manifestations in the oral cavity include pain, swelling, teeth displacement, twitching, paresthesia, and nasal obstruction. This paper presents an advanced chondroblastic osteosarcoma in the maxilla. Histological examination showed extensive cartilaginous neoplastic proliferation, with small areas in which osteoid formation was identified and non-lamellar bone, surrounded by neoplastic cells. The treatment consisted in maxillectomy and bilateral neck dissection. In addition, sealing stents were placed to seal the defect orosinonasal communication. Although osteosarcoma of the jaw has a better prognosis than osteosarcoma of long bones, jaw osteosarcoma patients with advanced tumors can occur, especially when the proper diagnosis is not appropriate.

Key words: Osteosarcoma, osteogenic sarcoma, bone tumor.

Fecha de recepción: 17 de febrero de 2011.

Aceptado para publicación: 28 de febrero de 2011.

Álvarez Martínez E, Castro Espitia L, Ardila Medina CM. Osteosarcoma condroblástico maxilar: Presentación de un caso. Av. Odontoestomatol 2012; 28 (5): 227-231.

* Cirujano Maxilofacial. Profesor Titular Facultad de Odontología Universidad de Antioquía.

** Estudiante postgrado Cirugía Oral y Maxilofacial. Universidad de Antioquía.

*** Profesor Asociado. Director posgrado de Periodoncia. Facultad de Odontología. Universidad de Antioquía. Director del Grupo de Estomatología Biomédica. Universidad de Antioquía. 


\section{INTRODUCCIÓN}

Los osteosarcomas son neoplasmas caracterizados por la presencia de células mesenquimales que producen tejido osteoide (1). Se observa regularmente en niños y adultos jóvenes, con discreto predominio en los hombres (2), y una incidencia anual de una por cien mil personas (3). El osteosarcoma de los maxilares (OM) representa solamente el $7 \%$ de todos los sarcomas osteogénicos (2) afectando con igual frecuencia el maxilar y la mandíbula (4). El osteosarcoma condroblástico es uno de los subtipos de osteosarcoma que se caracteriza por una diferenciación cartilaginosa que raramente afecta la región maxilofacial.

Las manifestaciones clínicas en la cavidad bucal comprenden dolor, inflamación, desplazamiento y/o pérdida de los dientes asociados, espasmos, parestesias y obstrucción nasal (4). La lesión puede ser radio lúcida, radiopaca, o una combinación de las dos, con márgenes irregulares pobremente definidos $(2,4)$.

Los protocolos de tratamiento para el osteosarcoma incluyen cirugías radicales o conservadoras, complementadas con radioterapia y/o quimioterapia (2). Este artículo presenta las características clínicas e histológicas y el tratamiento de un osteosarcoma condroblástico localizado en el maxilar superior.

\section{CASO CLÍNICO}

En agosto de 2008, un paciente de 20 años de edad y sexo masculino, consultó al odontólogo general por presencia de una masa de $3 \mathrm{~cm}$ de diámetro en el paladar, no dolorosa, indurada, pétrea, asociada a movilidad del incisivo central superior derecho. En ese momento se le realizó radiografía panorámica (Figura 1) y biopsia. El diagnostico histológico indicó la presencia de un tumor condroide compatible con condrosarcoma mesenquimal. Las tinciones inmunohistoquímicas revelaron negatividad para P63, Cam 5, 2 y actina de músculo liso. El índice de proliferación celular KI 67 fue del 10\%. El tratamiento consistió en la escisión quirúrgica de la masa.

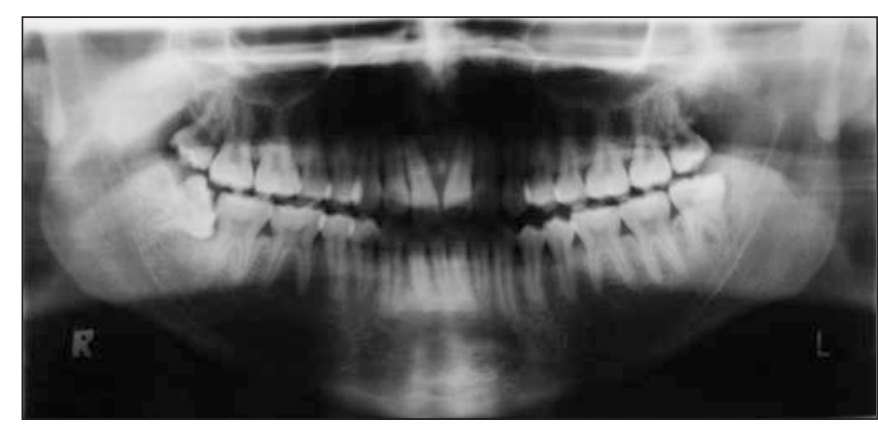

Fig. 1. Radiografía Panorámica que presenta el aspecto de la lesión en el año 2008.

Después de un año y medio, el sujeto presentó un aumento enorme del tumor y se remitió a la Unidad de Cirugía Oral y Maxilofacial del Hospital Universitario San Vicente de Paúl de la ciudad de Medellín, Colombia. En el momento del ingreso, se observó una gran masa submucosa de aspecto necrótico (Figura 2), con compromiso del maxilar, perdida de varios dientes y linfadenopatía cervical. Se ordenó tomografía computarizada de cara y cuello, con medio de contraste, en la cual se visualizó la enorme patología. El examen histológico evidenció proliferación neoplásica extensamente cartilaginosa,

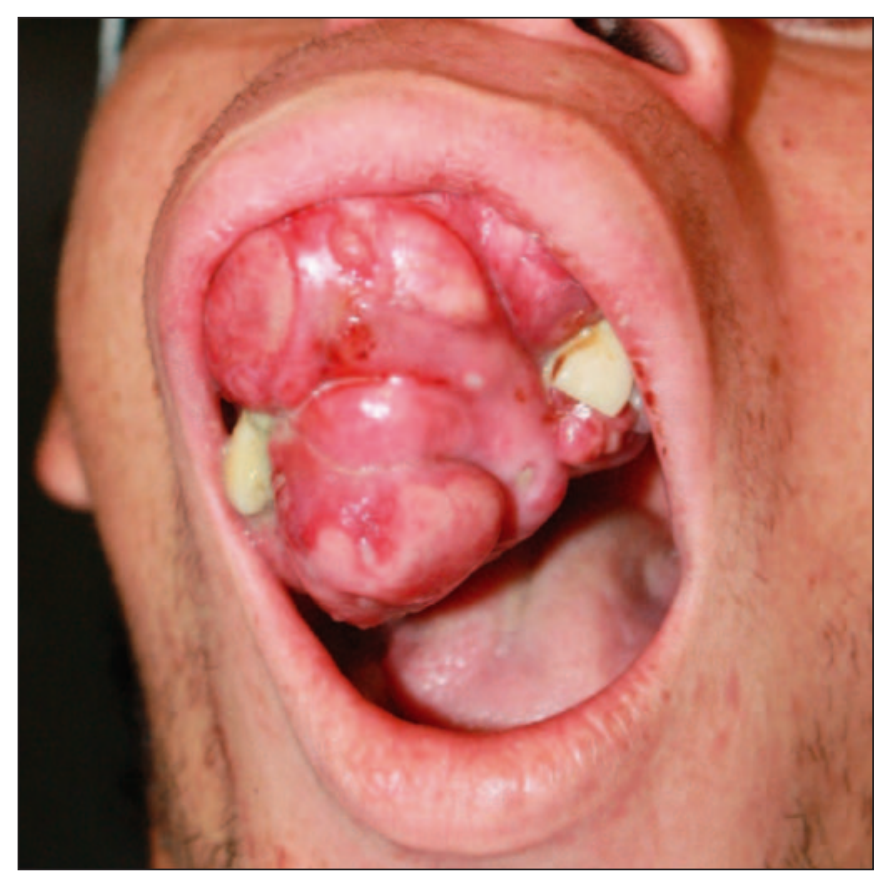

Fig. 2. Aspecto de la lesión tumoral en el año 2010. 
con áreas pequeñas en la que se identificó formación osteoide y hueso no laminar, rodeado por células neoplásicas. Adicionalmente, se observó diferenciación osteoblástica, áreas con pleomorfismo nuclear, núcleos atípicos y necrosis (Figura 3). El diagnóstico definitivo fue osteosarcoma condroblástico maxilar.

El tratamiento consistió en maxilectomía y vaciamiento bilateral de cuello (Figuras 4 y 5). Se colocó férula quirúrgica obturadora (Figura 6) con el fin de sellar

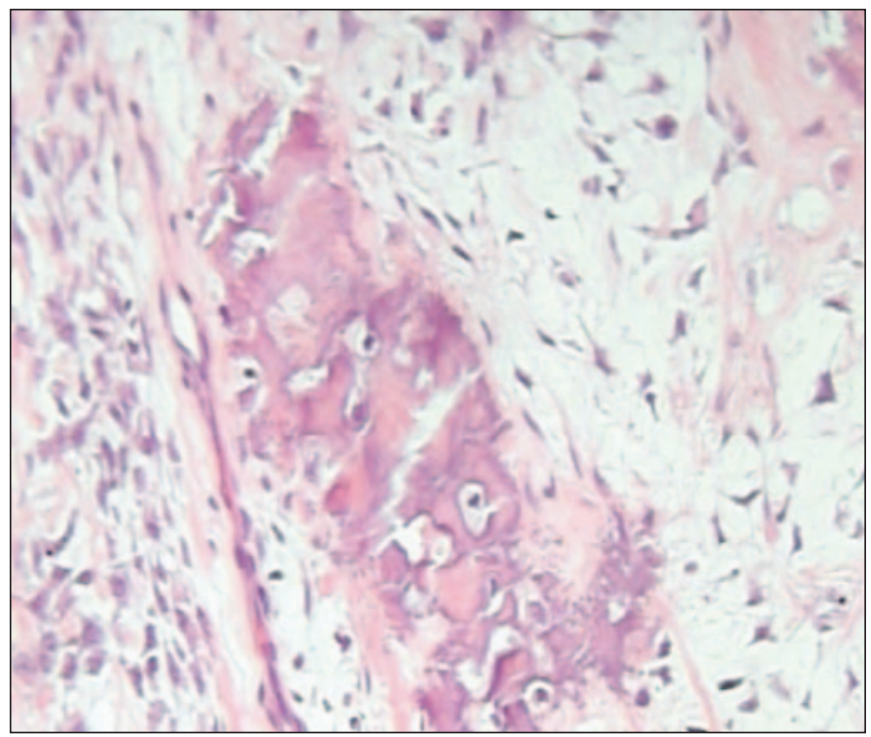

Fig. 3. Placa histopatológica.

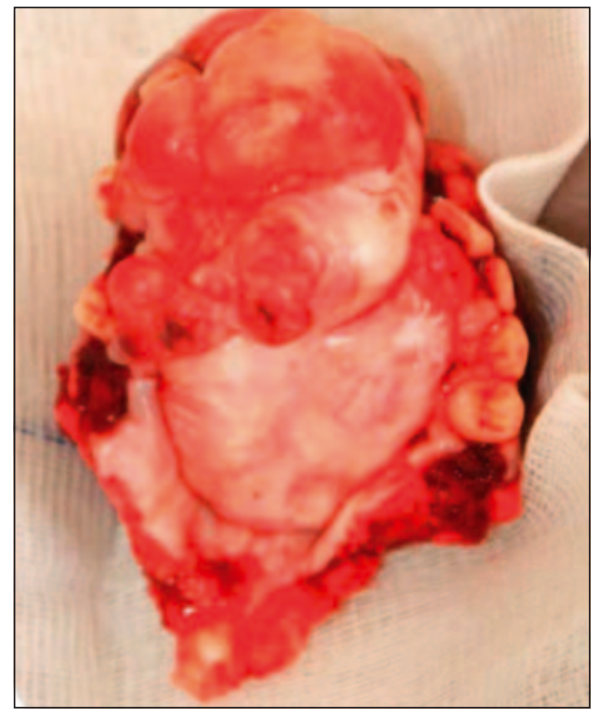

Fig. 4. Maxilectomía.

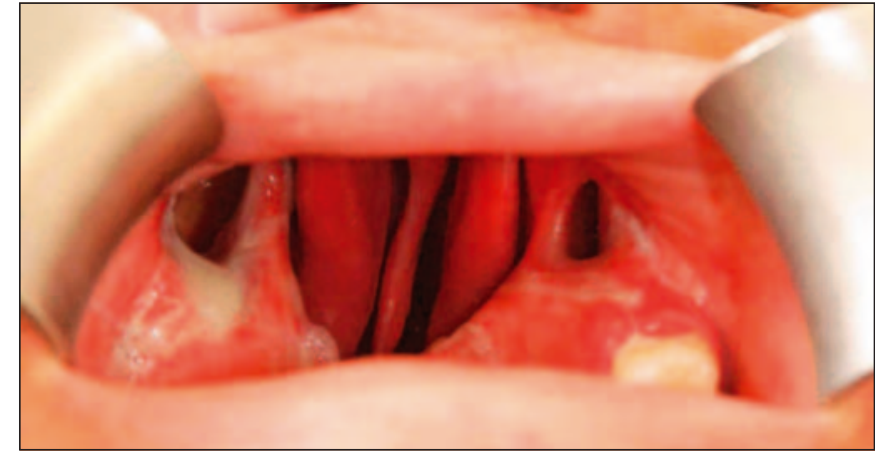

Fig. 5. Posquirúrgico inmediato.

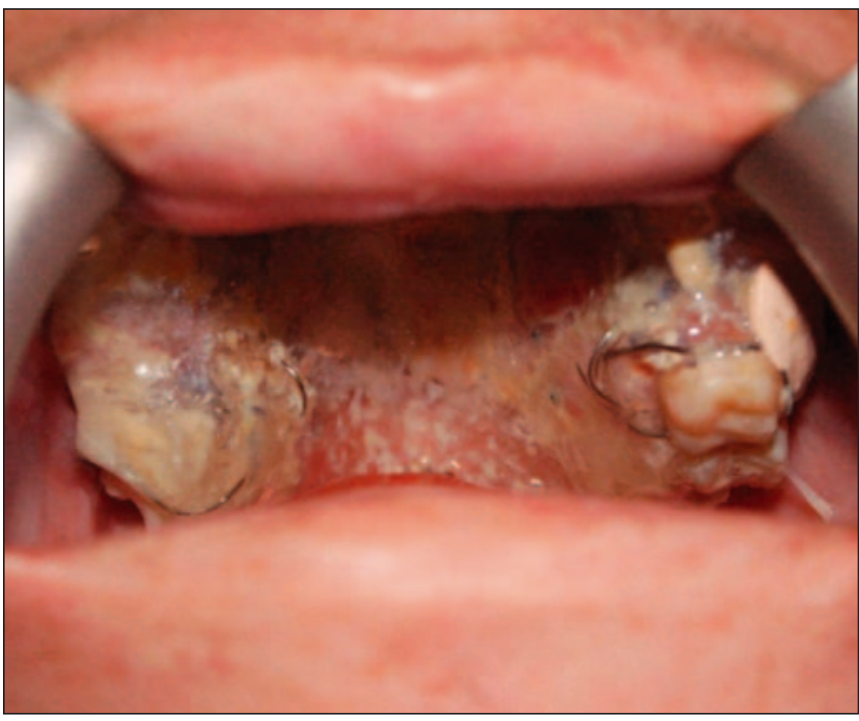

Fig. 6. Férula obturadora.

el defecto de la comunicación oronasosinusal. Posteriormente, se tomó impresión para confección de somatoprotesis.

Treinta días después de la resección, se programaron 35 sesiones de radioterapia. Seis meses más tarde, se realizó control clínico y tomográfico, (Figura 7) en donde no se identificó recidiva de la patología.

\section{DISCUSIÓN}

El OM es el osteosarcoma más frecuente de cabeza y cuello, aun cuando son tumores de muy baja inci- 


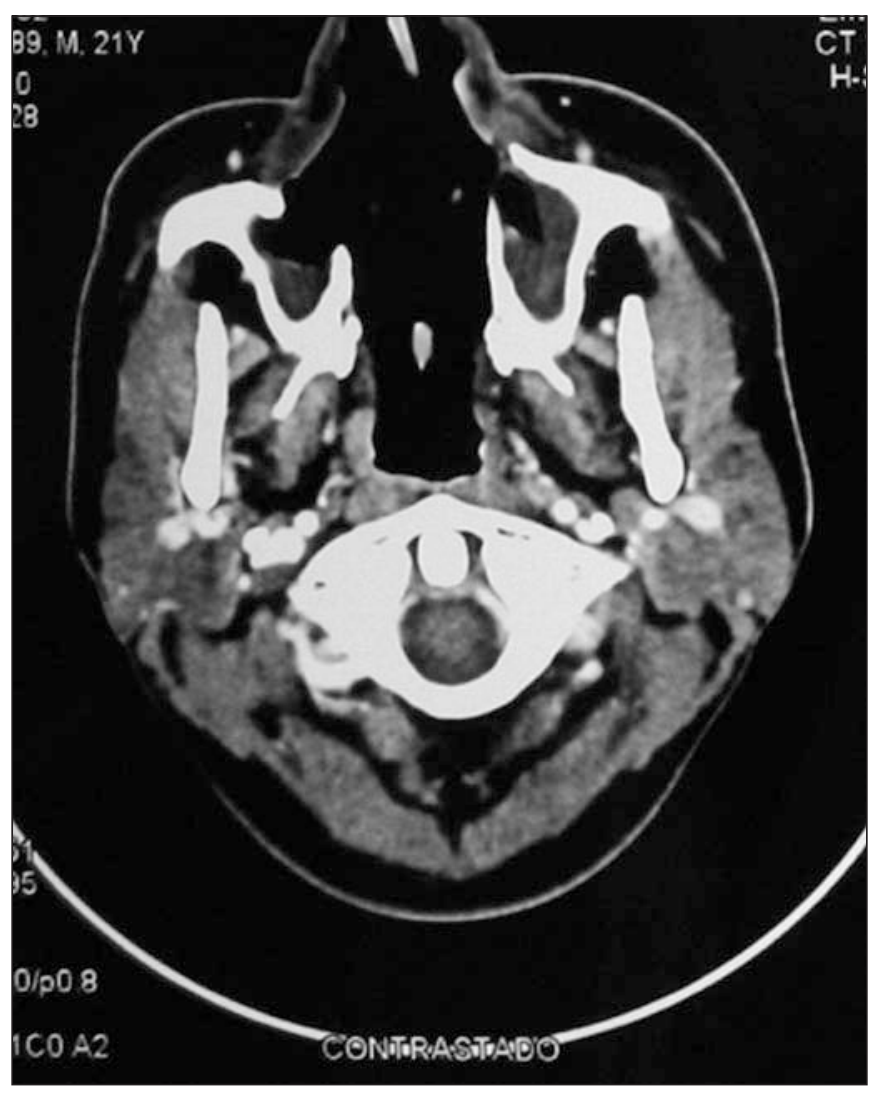

Fig. 7. Control posquirúrgico a los seis meses.

dencia (3). La etiología del osteosarcoma es desconocida, sin embargo causas secundarias a esta patología incluyen radiación ionizante, retinoblastoma hereditario, enfermedad de Paget, condromatosis, exostosis hereditaria múltiple y displasia fibrosa (5).

Las particularidades clínicas del OM, la edad y sexo del paciente del presente caso, concuerdan con las características informadas frecuentemente en diferentes estudios $(3,4)$. Sin embargo, se ha notado una distribución por edad en dos diferentes etapas de la vida: entre 10 y 20 años, o entre la quinta y la sexta década (6).

En el presente caso no se observó metástasis del tumor, no obstante, solamente el $8 \%$ de los pacientes no la presentan (6). La metástasis se observa con mayor regularidad en el pulmón (90\%) y los huesos (10\%) (7).

Histológicamente, el osteosarcoma es un neoplasma que produce tejido osteoide (2, 3). Dependiendo del tipo de matriz predominante dentro del tumor, los cuatro subtipos histológicos incluyen las formas osteoblástica, fibroblástica, condroblástica y telangiectásica. El pronóstico de los cuatro es similar (8), aun cuando el condroblástico se presenta con mayor frecuencia en los maxilares (9). En el presente caso, la proliferación neoplásica extensamente cartilaginosa, con áreas pequeñas en la que se identificó formación osteoide, confirmaron el diagnóstico de osteosarcoma condroblástico.

Siguiendo las recomendaciones de la literatura científica $(8,9)$, el OM se trató por medio de una resección quirúrgica. En casos avanzados, la radioterapia o quimioterapia puede usarse en asociación con el tratamiento quirúrgico, o como terapia paliativa (8). En este caso se realizó una resección quirúrgica con amplios bordes negativos, con el fin de mejorar el pronóstico de la lesión (9). Aun cuando el OM presenta un mejor pronóstico que el osteosarcoma de los huesos largos, los pacientes con OM pueden presentar tumores avanzados, principalmente cuando no se realiza el diagnóstico adecuado (10).

\section{BIBLIOGRAFÍA}

1. Sandberg AA, Meloni-Ehrig AM. Cytogenetics and genetics of human cancer: methods and accomplishments. Cancer Genet Cytogenet. 2010; 203:102-26.

2. Cabral LA, Werkman C, Brandão AA, Almeida JD. Imprint cytology of osteosarcoma of the jaw: a case report. J Med Case Reports. 2009 11;3: 9327.

3. Sturgis EM, Potter BO. Sarcomas of the head and neck region. Curr Opin Oncol. 2003;15:23952.

4. Ogunlewe MO, Ajayi OF, Adeyemo WL, Ladeinde AL, James O. Osteogenic sarcoma of the jaw bones: a single institution experience over a 21year period. Oral Surg Oral Med Oral Pathol Oral Radiol Endod. 2006;101:76-81.

5. Bramwell VH. Osteosarcomas and other cancers of bone. Curr Opin Oncol. 2000;12:330-6. 
6. Marina N, Gebhardt M, Teot L, Gorlick R. Biology and therapeutic advances for pediatric osteosarcoma. Oncologist 2004;9:422-41.

7. Ahn S, Choi JA, Chung JH, Choi H, Chun EJ, Choi SI, et al. MR imaging findings of a primary cardiac osteosarcoma and its bone metastasis with histopathologic correlation. Korean J Radiol. 2011;12:135-9.

8. Tan JZ, Schlicht SM, Powell GJ, Thomas D, Slavin JL, Smith PJ, et al. Multidisciplinary approach to diagnosis and management of osteosarcoma - a review of the St Vincent's Hospital experience. Int Semin Surg Oncol. 2006;3:38.

9. McGuff HS, Heim-Hall J, Holsinger FC, Jones AA, O'Dell DS, Hafemeister AC. Maxillary osteo- sarcoma associated with a dental implant: report of a case and review of the literature regarding implant-related sarcomas. J Am Dent Assoc. 2008; 139:1052-9.

10. Amaral MB, Buchholz I, Freire-Maia B, Reher P, de Souza PE, Marigo Hde A, et al. Advanced osteosarcoma of the maxilla: a case report. Med Oral Patol Oral Cir Bucal. 2008;13:E492-5.

\section{CORRESPONDENCIA}

Carlos M. Ardila M

Calle 64 No $52-59$

Medellín, Colombia.

E-mail: martinardila@gmail.com 